\title{
La vivienda adecuada financiarizada según el ingreso. El discurso de las Naciones Unidas
}

\author{
Adequate financialised housing according to household income. \\ The United Nations discourse
}

\section{Mónica Mejía-Escalante}

Monica Mejía-Escalante

Universidad Nacional de Colombia, Sede Medellín (Colombia)

Facultad de Arquitectura Escuela del Hábitat Grupo de Investigación Escuela del Hábitat

Magíster en Hábitat, Universidad Nacional de Colombia Sede Medellín (Colombia).

Doutorado em Arquitetura e Urbanismo, Universidade de São

Paulo (Brasil).

Par evaluadora e investigadora junior reconocida por Colciencias (2018-2020).

Coordinadora, Maestría en Hábitat Universidad Nacional de Colombia sede Medellín.

Profesora, Universidad Nacional de Colombia sede Medellín,

Facultad de Arquitectura, Escuela del Hábitat-Cehap.

https://scienti.minciencias.gov.co/cvlac/visualizador/generarCurriculoCv.do?cod rh $=000128 \mid 364$

https://scholar.google.es/citations?hl=es\&user=t\&pli= I \&u-

ser $=$ tFz8pt8AAAAJorcid.org/0000-0002-2768-I550

(D) orcid.org/0000-0002-2768-1550.

@memejiae@unal.edu.co

Mejía-Escalante, M. (202I). La vivienda adecuada financiarizada según el $\vdots$ ingreso. El discurso de las Naciones Unidas. Revista de Arquitectura (Bogotá),

43-53. https://doi.org//0.14718/RevArq.2021.3330

23(I),

\section{Resumen}

El acceso a la vivienda adecuada se instaura según el ingreso familiar y mediante mecanismos de financiación desde la década de 1940. El presente texto examina la evolución de la noción vivienda adecuada en textos de las Naciones Unidas entre 1946 y 2020, a partir de los cuales se hace un análisis de contenido que se pone en relación con las variables habitacionales que 21 países de América Latina y el Caribe aplican para sus diagnósticos residenciales sobre vivienda adecuada, y con la cualificación dada al derecho a la vivienda en sus constituciones, cuyo componente objetivo son los indicadores habitacionales que las naciones aplican. Nociones equivalentes como vivienda digna, económica, saludable y hábitat son construidas a manera de lema por las Naciones Unidas, y desde ellas se instala la vivienda financiarizada, que no se asimila a la vivienda adecuada. Las inadecuaciones en vivienda, entre las cuales se encuentran los instrumentos de financiación para su acceso, perturban la salud del morador; esta apreciación podría potenciar la comprensión de la vivienda como abrigo en la garantía de los derechos habitacionales, y no solo como objeto de riqueza; en particular, en momentos de excepción como los que surgen con una pandemia.

Palabras clave: Vivienda financiarizada; hábitat; indicadores habitacionales; derecho a la vivienda; urbanización

\section{Abstract}

Access to adequate housing has been established according to family income and through financing mechanisms since the 1940s. This text examines the evolution of the notion of "adequate housing" in United Nations texts between 1946 and 2020, from which a content analysis is carried out in relation to the housing variables that twenty-one Latin America and Caribbean countries apply for their residential diagnoses on adequate housing, and with the qualification given to the right to housing in their constitutions, whose objective component are the housing indicators that the nations apply. Equivalent notions such as decent, affordable, healthy housing and habitat are constructed as a motto by the United Nations, from which finiancialization of housing is installed, which is not assimilated to adequate housing. The inadequacies in housing, among which are the financing instruments for its access, disturb the health of the dweller. This appreciation could enhance the understanding of housing as a shelter in the guarantee of housing rights and not only as an object of wealth, particularly in times of exception such as those that arise with a pandemic.

Keywords: Finiancialization of housing; habitat; housing needs; right to housing; urbanization

\section{Introducción}

Esta investigación hace parte de tesis de Doctorado en Arquitetura e Urbanismo, de la Universidade de São Paulo (Brasil), con el título Moradia adequada e dignidade humana na experiência espacial (2016). El doctorado en mención es desarrollado dentro del marco de una comisión de estudios para doctorado de la Universidad Nacional de Colombia (2012-2016), financiado con el Programa Crédito Beca Colfuturo Colombia (2012-2016) y Beca 679 Doctorados en el exterior, de Colciencias (2014-2016).

El acceso a la vivienda adecuada se instaura según el ingreso familiar y mediante mecanismos de financiación desde la década de 1940, por parte de las Naciones Unidas, que es la fuente institucional que da origen a las directrices sobre asentamientos humanos para los países que la integran. Contribuye a este escenario que el indicador del Derecho a la Vivienda Adecuada, del Pacto Internacional de Derechos Económicos, Sociales y Culturales (PIDESC), de 1991, diagnostica áreas residenciales en el mundo como adecuadas o inadecuadas, y a sus pobladores, en franjas estratificadas de ingreso económico, para mostrar una vivienda financiarizada que no siempre es adecuada; es decir, se halla atravesada por instrumentos de financiación en su acceso y su provisión, así como en la garantía de su derecho, pasando por la cotidianidad en el residir. 
El examen de los asuntos epidemiológicos en el medio humano, observado desde criterios de la Organización Mundial de la Salud (OMS), posibilita indagar por las perturbaciones en la salud del morador causadas por las inadecuaciones en la vivienda, que, además, envuelven las medidas de financiación para su acceso. Esta apreciación aportaría información para comprender la vivienda como abrigo, y no solo como objeto de riqueza en la garantía de los derechos habitacionales; sobre todo, en momentos de excepción como los que surgen con una pandemia.

Los documentos de las Naciones Unidas (en concreto, las resoluciones — son recomendaciones que buscan instituir criterios para legislar las prácticas de los países-) develan que el discurso sobre la vivienda se estructura a partir de: 1) un problema construido respecto a la vivienda y los asentamientos humanos; 2) una noción equivalente a la vivienda adecuada, que, a manera de lema, denota el problema que debe ser solucionado por las Naciones Unidas; 3) programas de acción para resolver el problema de vivienda; 4) la vigencia de la noción equivalente o el periodo en el que los programas de acción deben ser aplicados para solventar el problema; 5) el modo de medición del problema desde los indicadores construidos por cuerpos anexos a las Naciones Unidas, estipulados como universales y que las naciones integrantes acogen, y 6) el medio de difusión de los diagnósticos residenciales en informes globales a partir de los indicadores universales. Dicha estructura se encuentra evidenciada en los subtítulos de la presente introducción y en la figura 2.

La evolución de la provisión de vivienda adecuada desde el discurso de las Naciones Unidas se detalla en este texto a manera de recortes temporales entre 1946 y 2020, desde las nociones análogas a la vivienda adecuada descubiertas como lema, y mostradas en los subtítulos entre comillas, acompañadas del programa de acción, su vigencia entre paréntesis, y el medio de difusión que corresponde a los protocolos de derecho internacional y las conferencias mundiales.

La provisión de vivienda por parte de los Estados en Europa se desarrolla a fines del siglo XIX e inicios del XX, como respuesta a la situación social de los barrios precarios, carentes de condiciones de saneamiento, como resultado de la urbanización y su creciente población, pues hasta entonces la vivienda era un asunto de aprovisionamiento particular, y no materia de regulación nacional e internacional; también, por los movimientos sociales que exigían mejores condiciones en los lugares de trabajo (Craven, 2003). Se han observado dos formas de pensar el problema de la vivienda desde mediados de la primera mitad del siglo XX: 1) según la eficacia funcional de la vivienda, un good, un bien de valor económico que provee beneficios y lo convierte en una variedad de actividades legales, y 2) según el valor intrínseco de la vivienda, que es el abrigo, y que conduciría a la preservación de la dignidad humana y la supervivencia, un concepto ligado más a la idea de home que de house (Craven, 2003).

\section{Enfrentar el "alojamiento de emergencia" en Europa. Diagnóstico sobre la vivienda en informes mundiales (1946-1960)}

Las Naciones Unidas inician el interés por la vivienda con la Resolución 53 de la Asamblea General (1946), titulada Vivienda y Urbanismo, y cuyo problema era enfrentar el "alojamiento de emergencia" como noción análoga a la vivienda adecuada, en una Europa arrasada por la guerra, bajo la premisa de construcción de habitaciones económicas y rápidas. Sin diagnósticos, en esta resolución se recomienda al Consejo Económico y Social (Ecosoc) desarrollar estudios para enfrentar el "alojamiento de emergencia", correspondiente, en su mayoría, a naciones de Europa y unas pocas de otros continentes, que se difunde en los Informes sobre la Situación Social en el Mundo de 1952 y de 1961 al respecto de: 1) viviendas disponibles y futuras necesidades habitacionales; 2) metodologías para la construcción de vivienda pública; 3) financiación de la vivienda; 4) métodos para incrementar la rapidez en la construcción de domicilios; 5) problemas sociales, económicos y tecnológicos en la planeación de áreas urbanas y rurales; 6) conjuntos de vivienda, y 7) educación de los planificadores. ${ }^{1}$

\section{La "vivienda económica y servicios comunales conexos". Financiación externa, asistencia técnica, autoconstrucción y otras formas de asociación desde las políticas de vivienda nacionales (1952-1975)}

El acceso a la "vivienda económica y servicios comunales conexos", de la Asamblea General de las Naciones Unidas (1960), es el problema por solucionar en las décadas de 1950 y 1960. La migración de la población de áreas rurales a las ciudades y la consecuente insuficiencia de servicios urbanos, además de la dificultad de la población rural para adaptarse a la nueva vida, es lo que la Asamblea General (1961) denomina urbanización; no obstante, la Organización de las Naciones Unidas (ONU) consideró que el proceso de migración hacia las ciudades con ser-

\footnotetext{
1 Años de publicación de los Informes sobre la situación socia en el mundo: 1952, 1961, 1963, 1967, 1970, 1974, 1985 y 1989. A partir de 1990 se constituye en la serie Informe Global Sobre Asentamientos Humanos (Global Report on Human Settlements).
} 
vicios cesaría en la década de 1970; sin embargo, tal situación sigue sucediendo, más de medio siglo después, en todos los países del mundo.

Los "servicios comunales conexos" a una vivienda económica, tales como agua potable, energía y eliminación de excretas y residuos para las áreas residenciales emergentes, es el objetivo establecido para mitigar la escasez de viviendas y hacer frente a la urbanización, por lo cual se insta a que los gobiernos acepten financiación externa, construcción apoyada por asistencia técnica, y que los procesos constructivos permitan la tipificación y la prefabricación, además de la autoconstrucción, como forma de desarrollo urbano.

Bajo estos programas de acción para proveer habitación, los Estados asumen la función de permitir que se construyan y se vendan viviendas, sin comprender el abrigo como valor intrínseco de estas. Por dicha vía, a inicios del siglo XX en Europa, según Lefebvre (1983), comienza a instaurarse en las ciudades el pensamiento del capitalismo, el cual tiene interés en la división y la venta de la tierra por diversos actores como práctica de desarrollo urbano y como actividad permitida por las autoridades.

Empieza, también en palabras de dicho autor, a institucionalizarse la marginalización del habitar, con los nuevos barrios autosuficientes, o "suburbios", construidos bajo los principios del urbanismo de la Carta de Atenas de 1933: habitar, trabajar, recrearse y movilizarse (Le Corbusier, 1993). Lefebvre continúa manifestando que estos nuevos barrios fueron destinados, en principio, a los trabajadores, a los asalariados, a quienes se les buscaba otra función aparte de la de producir: la de ser propietarios, cuyo medio era una vida cotidiana basada en los principios del urbanismo; por tal razón, en estos barrios de vivienda económica y servicios conexos el valor de uso —el habitar, la vida y el tiempo urbanose separa del valor de cambio —el consumo de productos, bienes y servicios- (Lefebvre, 1983).

\section{De la "vivienda como componente del derecho a un nivel de vida adecuado" al "derecho a la vivienda adecuada". Declaración Universal de los Derechos Humanos (1948) y Pacto Internacional de Derechos Económicos, Sociales y Culturales (1966; 1991)}

La Declaración Universal de los Derechos Humanos (DUDH) instituye componentes del derecho al nivel de vida, "la salud y el bienestar, y en especial la alimentación, el vestido, la vivienda, la asistencia médica y los servicios sociales necesarios" (Asamblea General de las Naciones Unidas, 1948, p. 7). Como desde la década de
1950 el enfoque era la vivienda económica cualificada por servicios conexos, el acceso a los componentes del nivel de vida —entre ellos, la vivienda-, enunciados en la DUDH de 1948, se alcanza conforme la capacidad económica del habitante, que es definida por su salario en el artículo 23 de la declaración: "Toda persona que trabaja tiene derecho a una remuneración equitativa y satisfactoria, que le asegure, así como a su familia, una existencia conforme a la dignidad humana" (Asamblea General de las Naciones Unidas, 1948, p. 7).

La remuneración por un trabajo se refiere al salario real que, según la Academia de Ciencias de la Unión Soviética, es expresado en componentes de subsistencia para el trabajador, y que indica cuáles ítems de los componentes del nivel de vida adecuado puede adquirir con el monto de su sueldo. Así, la magnitud del salario real es determinada por los precios de los ítems de consumo y servicios, por los costos de los alquileres y por el volumen de impuestos cobrados al trabajador, de manera que una gran fracción del salario ha sido considerada un sistema de pago en especie entregado al trabajador, lo cual consiste en que a este se le proporciona un lugar para vivir o para dormir, alimentación y algún dinero, y así el capitalista puede explotarlo como vendedor de fuerza de trabajo y como consumidor (Academia de Ciencias de la Unión Soviética, 1967). El pago en especie remite a los componentes del nivel de vida expuestos en la DUDH de 1948.

El secretario general de las Naciones Unidas, en cooperación con la Organización Internacional del Trabajo (OIT) y otros cuerpos de las Naciones Unidas, busca instaurar, desde una guía, mínimos esenciales mediante variables para los componentes del nivel de vida, "y se propuso expresar estos componentes por medio de medidas o 'indicadores' estadísticos concretos" (Organización de las Naciones Unidas, 1961, p. 1). El documento guía fija una diferencia entre nivel de vida, estándar y norma estableciendo el nivel de vida como las condiciones actuales en las que vive un pueblo; el estándar de vida, como las condiciones que se consideran adecuadas y apropiadas para una u otra población, y la norma, como la condición deseable definida por y para propósitos particulares (Organización de las Naciones Unidas, 1961).

Para la vivienda, las variables se corresponden con lo establecido por la OMS (1961) en su documento sobre criterios de salubridad en la vivienda y el medio humano, y cuyo fin es el cuidado de la integridad física y mental del hombre. Las condiciones cualitativas de habitación fueron abordadas con más detalle en la Recomendación sobre la Vivienda de los Trabajadores número 115 de la OIT (1961), a modo de lineamientos espaciales para los domicilios y las áreas para 
dormir, que eran entregados al trabajador como salario en especie. Las variables para el componente vivienda son:

Porcentaje de la población que habita en "viviendas";

1) Porcentaje de viviendas ocupadas en las que haya tres o más personas por habitación;

2) Porcentaje de viviendas ocupadas que tienen agua corriente dentro de la vivienda o fuera de ella, pero a no más de cien metros;

3) Porcentaje de viviendas ocupadas con excusado. [...]

Indicadores suplementarios:

1. Porcentaje de la población que ocupa viviendas que no reúne las debidas condiciones de habitabilidad clasificadas como 'rústicas', 'improvisadas' y 'no aptas para ser habitadas', o que carecen de todo abrigo;

2. Promedio de personas por habitación (solo para las viviendas ocupadas);

3. Porcentaje de viviendas ocupadas dotadas de retretes inodoros (urbanas);

4. Porcentaje de viviendas ocupadas provistas de otro tipo de retrete. (Organización de las Naciones Unidas, 1961, p. 13)

La vivienda adjetivada como adecuada, como recurso para lograr el derecho a un nivel de vida digno, se establece desde el PIDESC de 1966 de la Asamblea General de las Naciones Unidas (1966); este enfatiza que las condiciones apropiadas en el trabajo y en el medio humano son promoción de la salud humana, mas no las vincula con la vivienda, aun cuando la relación entre salud y habitación ya se había concertado desde los indicadores prioritarios para la vivienda en la Guía de las Naciones Unidas de 1961, y desde convenciones sanitarias de la OMS sobre salubridad en el medio residencial.

El artículo 11 del draft del PIDESC de 1966 construido por la General Assembly (1955), insta a que desde este pacto se establezcan "todas las medidas necesarias", tales como legislación, subsidios, exenciones fiscales, préstamos y suministro de materiales en condiciones favorables para la población, para que la vivienda se viabilice como un servicio, con provisión y acceso a partir de mecanismos financieros, lo cual está en correspondencia con la vivienda económica y los servicios conexos con acceso desde un salario.

Así pues, el PIDESC de 1966 no delimita la vivienda como un derecho, el cual, por fin, se establece en el PIDESC de 1991. La Observación No. 7 del PIDESC del Comité de Derechos Económicos, Sociales y Culturales de (1997) aborda el tema de los desalojos forzosos como situación que debe ser atendida desde el derecho a la vivienda adecuada.
La vivienda adecuada significa disponer de un lugar donde poderse aislar si se desea, espacio adecuado, seguridad adecuada, iluminación y ventilación adecuadas, una infraestructura básica adecuada y una situación adecuada en relación con el trabajo y los servicios básicos, todo ello a un costo razonable. [...] [Los siete elementos son:]

1. Seguridad jurídica de la tenencia;

2. disponibilidad de servicios, materiales, facilidades e infraestructura;

3. gastos soportables;

4. habitabilidad;

5. asequibilidad;

6. lugar;

7. adecuación cultural. (Comité de Derechos Económicos, Sociales y Culturales, 1991, p. 2-4).

\section{Desde "sin hambre en el mundo" hasta "ciudades sin barrios de tugurios".} Decenios para el Desarrollo (1960-2000), Declaración del Milenio (2000-2015) y Objetivos de Desarrollo Sostenible (2015-2030)

En los inicios de la década de 1960 la preocupación emergente en materia de desarrollo era luchar contra el hambre en el mundo. La Organización de las Naciones Unidas para la Agricultura y la Alimentación (en inglés, FAO, por las iniciales de Food and Agriculture Organization) lidera los esfuerzos internacionales para apoyar a los países en dicha situación, con el lanzamiento, en 1960, de la Campaña Mundial contra el Hambre. Reconociendo que el problema no se reducía simplemente a este tema, se proclama el primer Decenio de las Naciones Unidas para el Desarrollo entre 1961-1970, para luego establecer consecutivamente tres decenios, más hasta la década de 2000. Debido a que a lo largo de cada decenio había un moderado progreso en el logro de los objetivos, se evidenciaban problemas de acceso y provisión a la vivienda económica desde instrumentos financieros, además de la degradación de amplias áreas residenciales en el mundo; ante ello, se instó a la Declaración del Milenio (ODM) en 2000, con 8 objetivos que lograr para 2015, a fin de mejorar significativamente la vida de por lo menos 100 millones de personas que vivían en asentamientos inadecuados. Así se establece el indicador de familia en asentamiento precario cuando esta carece de 2 o más de las siguientes condiciones: 1) vivienda con materiales durables, como protección contra vectores externos de enfermedades; 2) vivienda con no más de 2 personas por habitación; 3) acceso a agua potable; 4) vivienda con saneamiento y sanitario privado o colectivo, para un número razonable de personas, y 5) seguridad 
jurídica de la tenencia (Asamblea General de las Naciones Unidas, 2000).

Como el tiempo para cumplir los ODM era finito, se puso en marcha la Agenda 2030 para el Desarrollo Sostenible (ODS) con 17 objetivos y 169 metas, cuyo objetivo 11 busca que las ciudades y los asentamientos humanos sean "inclusivos, resilientes y sostenibles" para asegurar el acceso de todas las personas a la vivienda y a los servicios básicos adecuados y seguros, además de asequibles (Asamblea General de las Naciones Unidas, 2015). Los ODM-2015 y los ODS2030 reafirman la cualificación de lo adecuado de la vivienda desde la conexión a los servicios básicos y urbanos, como servicios a los que se puede acceder por medios económicos, mediante instrumentos financieros.

\section{De problemas del "medio humano" (1972) a problemas en el "medio ambiente" (1992) en los "asentamientos humanos". Conferencias mundiales Hábitat I (1976), Hábitat II (1996) y Hábitat III (2016)}

Asentamientos humanos, medio ambiente y hábitat fueron tres ámbitos que las Naciones Unidas expusieron en la década de 1970 con un común denominador: la vivienda. A fines de la década de 1960, esta misma organización se focaliza en detallar cómo las formas de habitar y la urbanización acelerada en los conglomerados urbanos en crecimiento —sobre todo, en los países en desarrollo- degradan la calidad del "medio humano"; esto motiva a que realice la Conferencia Mundial sobre el Medio Humano, en 1972, con principios comunes para guiar a las naciones hacia la preservación de este medio (Organización de las Naciones Unidas, 1972). La noción de "medio humano" fue reemplazada por la de "medio ambiente" en la Conferencia de las Naciones Unidas sobre el Medio Ambiente y el Desarrollo (CNUMAD), o Cumbre para la Tierra (Organización de las Naciones Unidas, 1992).

El Programa de las Naciones Unidas para los Asentamientos Humanos inicia en la década de 1970 con la Fundación Hábitat y el Programa de las Naciones Unidas para el Medio Ambiente (PNUMA); desde allí instauró, además de la Conferencia de 1972, la Conferencia de las Naciones Unidas sobre Asentamientos Humanos, más conocida como Hábitat I-1976 (Asamblea General de las Naciones Unidas, 1976). La Fundación Hábitat mudó su nombre a Centro de las Naciones Unidas para los Asentamientos Humanos (CNUAH), y 20 años más tarde las Naciones Unidas desarrolló la Segunda Conferencia de las Naciones Unidas para los Asentamientos Humanos Hábitat II-1996 sobre Vivienda Adecuada y Asentamientos Humanos (Asamblea General de las Naciones Unidas, 1996), y luego, Hábitat III-2016 sobre Vivienda Adecuada y Desarrollo Urbano Sostenible (Asamblea General de las Naciones Unidas, 2017).

La conferencia sobre el medio humano de 1972 se acerca al tema de la vivienda desde el control de recursos como agua, energía y residuos generados por los conglomerados humanos; es decir, servicios conexos a la vivienda. La conferencia sobre "medio ambiente" no focaliza su atención en los servicios conexos, sino en la influencia de los asentamientos humanos precarios, en la degradación del medio ambiente y en la gestión de los recursos naturales, así como en su demanda y su consumo. La vivienda es interpretada en Hábitat I-1976 como un componente del derecho a un nivel de vida adecuado; Hábitat II-1996 acoge la definición y los siete elementos del Derecho a la Vivienda Adecuada del PIDESC de 1991; Hábitat III-2016 delimita de nuevo a la vivienda como componente integrante del derecho a un nivel de vida adecuado y ratifica la realización progresiva del derecho a la vivienda a partir de mecanismos de financiación para su provisión y su acceso.

\section{De "enfermedades cuarentenables" a "cobertura sanitaria universal por pandemias" sin detrimento del tráfico mundial (1892- 2020)}

Catorce Conferencias Sanitarias Internacionales celebradas entre 1851 y 1938 bajo la gestión del gobierno francés, para estandarizar acciones a fin de detener la propagación de epidemias, bajo el precepto de "el mínimo de dificultad para el comercio con el máximo de protección en Salud Pública" (Mateos, 2005, p. 345), fueron de relevancia para la creación de la Office International d'Hygiene Publique (OIHP) fundada en 1907, y posteriormente dar paso a la OMS en 1946, en atención a problemas sanitarios relacionados con la vivienda y con el medio humano. La OMS expone en su documento sobre salubridad en áreas residenciales (1961) cómo el incumplimiento de los mínimos esenciales de los componentes del nivel de vida — en particular, los de la vivienda- pueden afectar la salud del morador, basándose en que la salud es un estado de completo bienestar físico, mental y social, y no solo la ausencia de afecciones o enfermedades (Organización Mundial de la Salud, 1946).

Los Principios de la Vivienda Saludable de 1990 se instauran en vínculo con el elemento habitabilidad del indicador Derecho a la Vivienda Adecuada del PIDESC de 1991, para que sean atendidos en correspondencia con la vivienda considerada una necesidad básica, que proporciona abrigo y protección; es decir, la vivienda 
home de Craven (2003). Estos principios son: 1) protección contra enfermedades transmisibles; 2) protección contra traumatismos, intoxicaciones y enfermedades crónicas; 3) reducción al mínimo de factores de estrés psicológico y social; 4) mejora del entorno habitacional; 5) uso adecuado de la vivienda, y 6) protección de poblaciones expuestas (Organización Mundial de la Salud, 1990).

La declaración de una pandemia mundial por parte de la OMS, en marzo de 2020, a causa del virus respiratorio SARS-CoV-2, y que inicia en China en 2019 y se expande a más de 150 países, obliga a que las naciones con contagios y muertes establezcan diversos niveles de confinamiento, cierre de fronteras y sectores durante más de 60 días en el primer semestre de dicho año. Esta organización, desde su Reglamento Sanitario Internacional (RSI, 2016) (cuyo objetivo es prevenir y controlar la propagación de enfermedades sin detrimento del tráfico internacional -es decir, en un contexto de mercado desde canales de financiación-), tiene estipulado desde principios del siglo XX medidas no farmacológicas, como el aislamiento domiciliario y el confinamiento social. Las órdenes para 2020, emitidas por diversos países, en el sentido de quedarse en casa para aislarse, trabajar y estudiar, así como para cuidar de los niños, los ancianos y los enfermos, y del aseo permanente y de buscar refugio para quien mora o trabaja informalmente en la calle, son difíciles de cumplir, si no imposibles, para un gran porcentaje de la población, no solo por la ausencia de viviendas seguras y adecuadas, sino por la falta de capacidad económica para su acceso.

Lo que se solicita en un confinamiento domiciliario y en un distanciamiento social es un comportamiento apropiado de los moradores en relación con las variables para la vivienda de la Guía de la Organización de las Naciones Unidas de (1961), de los Principios de la Vivienda Saludable de la OMS de (1990), del derecho a la vivienda adecuada del Comité de Derechos Económicos, Sociales y Culturales (1991), y de los ODM de la Asamblea General de las Naciones Unidas de (2000); pero la situación mundial en 2020 impacta los objetivos de estos indicadores. La Resolución A/75/148 de 2020 de la Asamblea General de las Naciones Unidas ha manifestado que en los países del mundo

[...] la COVID-19 ha sacado a la luz crisis ya existentes y ha generado otras nuevas. Ya antes de la pandemia de COVID-19 se había producido una pandemia de desalojos, aumento de la falta de hogar, existencia de viviendas inseguras o inadecuadas, discriminación en el acceso a la vivienda, aumento de los alquileres y especulación inmobiliaria y de tierras en demasiados países. (Asamblea General de las Naciones Unidas, 2020, p. 4)
Las mencionadas son situaciones que, según esta resolución, se exacerbaron con la pandemia, para llevar a una crisis mundial de salud pública y desempleo de proporciones aún insospechadas, además de profundizar las desigualdades socioeconómicas entre las líneas raciales, de género, de casta y de religión.

\section{Metodología}

El acceso a la vivienda adecuada según el ingreso familiar y mediante mecanismos de financiación se explora desde la década de 1940 como directriz por parte de las Naciones Unidas, en más de 9000 de sus resoluciones, sus instrumentos jurídicos internacionales, sus conferencias mundiales, sus declaraciones, sus convenciones y sus informes globales, para concretar la indagación desde el análisis de contenido en 300 documentos entre 1946, año de la primera resolución sobre vivienda de esta agencia, hasta noviembre de 2020, con un rastreo a partir de palabras clave en los títulos, como agua, albergue, asentamientos humanos, cooperación técnica, dignidad, derechos civiles y políticos, derechos económicos y sociales, energía, erradicación de la pobreza, estándar de vida, hábitat, medio ambiente, medio humano, microcrédito, planificación, urbanismo, urbanización, saneamiento, propiedad y vivienda Covid-19.

El levantamiento de los indicadores de déficit habitacional de 21 países de América Latina y el Caribe permitió comprender la base de sus diagnósticos residenciales y lo que constituye para estas naciones el grado de vivienda adecuada, y además, que se considera que sus variables son el componente objetivo de lo que se busca garantizar desde los derechos habitaciones. Se advierte que el origen de dichas variables es las Organización de las Naciones Unidas. Los indicadores habitacionales de la región son categorizados en déficit cuantitativo y cualitativo, examinados desde sus institutos de estadística que hospedan metodología para variables de vivienda y asentamientos humanos, y cuyos datos se observan en sus censos de población y vivienda y desde la encuesta de hogares realizada en los países hispanohablantes, con su equivalente en Brasil: la Pesquisa Nacional por Amostra de Domicílios (PNAD). Desde las constituciones vigentes de estas naciones se registraron las cualificaciones dadas al derecho a la vivienda, a la casa, al domicilio, a la habitación, al recinto, a habitação, moradia. La revisión de tan diversas fuentes y la profundidad en los datos, examinados desde el análisis de contenido, dificultó la consulta sobre el indicador habitacional de los países de la región. 


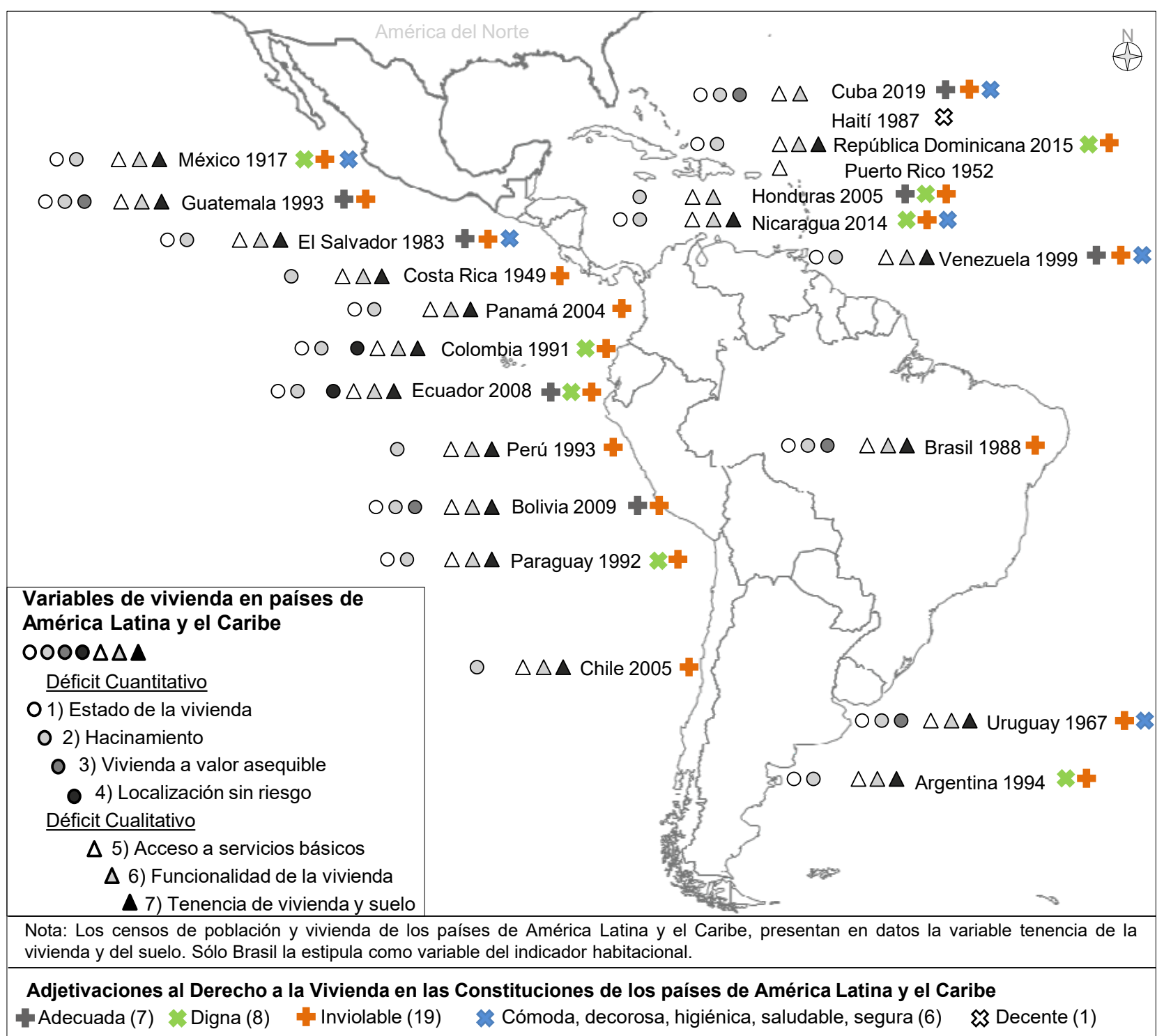

\section{Resultados}

La Guía de las Naciones Unidas de (1961) sobre los mínimos esenciales de los componentes del nivel de vida enuncia que siempre deberán ser completadas las estadísticas con material descriptivo, fundamentado en información regional, interpretando el concepto local de vivienda y, según cada cultura, evaluando el nivel y los servicios de cada comunidad y teniendo en cuenta la disponibilidad y la comparabilidad internacional de los datos. Tal información local no es diagnosticada para los países de América Latina y el Caribe (ALC), debido a que sus indicadores de déficit habitacional no posibilitan levantar datos particulares; además, como se observa en la figura 1, las variables son comunes para ALC, derivadas de los indicadores instituidos por las Naciones Unidas.

Las constituciones de los países de América Latina y el Caribe cualifican el derecho a la vivienda de diversas formas: adecuada, barata, cómoda, confortable, decente, decorosa, digna, de interés social, económica, inviolable, popular, que garantice la privacidad familiar, sagrada, con servicios básicos esenciales que incluyan un hábitat que humanice las relaciones familiares, vecinales y comunitarias, un direito social. Se advierte la poca coherencia de las variables en los indicadores de habitación de estas naciones con las adjetivaciones dadas a la vivienda como derecho en sus cartas políticas.

La provisión y el acceso a la vivienda financiarizada que las Naciones Unidas instaura mediante nociones equivalentes a la vivienda adecuada, a manera de lema e indicadores de habitación para diagnósticos residenciales, y su relación con la cualificación al derecho a la vivienda de los países de América Latina, se presentan en la figura 2, la cual se compone de dos líneas de tiempo: 1) en la parte superior, cuatro vertientes de acción: vivienda como servicio, a la vez que dentro de un marco de derechos; asentamientos humanos, medio ambiente y salud; y 2) la línea de tiempo concerniente a las constituciones de 21 países de América Latina y el Caribe (línea inferior). La vertiente de acción Salud ha estado presente con los asuntos epidemiológicos en el medio humano, desde que las Naciones Unidas inicia sus actividades, pero supeditada a llamar la atención sobre cómo las inadecuaciones en la vivienda, los asentamientos humanos y el medio ambiente pueden perturbar la integridad del hombre.

Cada vertiente de acción exhibe nociones equivalentes a la vivienda adecuada a modo de lema, el cual engloba el problema de habitación
(4) Figura 1. Variables de vivienda, adjetivación al derecho a la vivienda en las constituciones y año de la carta política de los países de América Latina y el Caribe. Fuente: elaboración propia (2020). 
para solucionar, según las Naciones Unidas, en un escenario donde la vivienda adecuada se comporta como un servicio atravesado por mecanismos de financiación, divulgado en protocolos y conferencias mundiales. Las nociones equivalentes a vivienda en cada una de las vertientes de acción se muestran en letra mayúscula y negrita, como se observa en la figura 2, y en los subtítulos de la introducción del artículo entre comillas; los programas de acción vigentes se enseñan bajo la noción análoga a vivienda adecuada. Las adjetivaciones a la vivienda como derecho en las constituciones de los 21 países de América Latina y el Caribe y el año de la constitución vigente son exhibidas en la línea de tiempo inferior. Entre las 2 líneas de tiempo se exponen los indicadores de vivienda y sus variables de habitación instituidos por las Naciones Unidas, aplicados desde el indicador de déficit habitacional de los países de América Latina y el Caribe como diagnósticos sobre vivienda adecuada, los cuales se difunden desde los protocolos y las conferencias mundiales de dicha organización, mostrados en la línea de tiempo superior.

Como producto de la crisis económica de la década de 1970 en Europa, el Banco Mundial (BM) aconseja a los gobiernos que cambien su rol anterior como productores de vivienda y pasen a ser facilitadores, para apoyar la expansión de mercados privados en este sector (Banco Mundial, 1993, p. 1). Rolnik (2015) afirma que en dicha década, a través de préstamos internacionales fueron financiados proyectos piloto de vivienda con servicios conexos en áreas residenciales precarias - particularmente, en América Latina-, para luego, en la década de 1980, abandonar progresivamente la financiación de estos proyectos urbanos. En esos momentos la política del BM se direcciona a realizar préstamos a instituciones financieras para crear condiciones y corporaciones destinadas a promover sistemas financieros habitacionales que viabilicen la compra de casa propia. En la década siguiente se concreta la creación de un sector financiero habitacional. En todas estas etapas, América Latina y el Caribe, según declara Rolnik (2015), se han mantenido como clientes de relevancia.

Así pues, los programas instaurados por las Naciones Unidas para hacer frente a la escasez de vivienda y a los problemas de la urbanización desde instrumentos de financiación para acceso y provisión de esta se practican en América Latina y el Caribe desde la década de 1950. Rolnik (2015) revela que siempre lo han hecho acompañados por la asistencia técnica de consultores de agencias como el BM, el Banco Central Europeo y otros bancos y organizaciones multilaterales, con tres instrumentos para viabilizar la demanda: 1) el acceso a la vivienda según el ingreso del habitante (el derecho de propiedad); 2) el desa- rrollo de un sistema financiero de vivienda, con la creación de instituciones de crédito hipotecario que acaparen demanda en todas las franjas de ingreso económico de la población, y 3) la racionalización focalizada de subvenciones para habitación por parte de los Estados. Este modelo es conocido desde el decenio de 1990 como grant subsidies (otorgamiento de subsidios), y es ampliamente reproducido en la región; principalmente, en Brasil, Bolivia, Colombia, Chile, Costa Rica, Ecuador, El Salvador, Guatemala, México, Panamá, Perú y Venezuela (2015).

Las áreas residenciales precarias también son alcanzadas por la mercantilización de la vivienda. Cuando UN-Hábitat lanza la política de mejora de asentamientos precarios en 2000, la solución se enfoca en asegurarles a estas familias acceso a financiamiento; el denominado microfinanciamiento habitacional para mejora de vivienda y servicios conexos, un modelo en el cual se presupone que la vivienda es un activo de inversión, para financiar el consumo, el bienestar o la actividad emprendedora (Rolnik, 2015), y para incluir a más población en el acceso a la vivienda mercantilizada, bajo el supuesto de mejorar las condiciones de vida, alcanzar una vivienda adecuada y combatir la pobreza.

\section{Discusión}

Pisarello (2003) expresa que todo derecho, por estar en la Constitución de un país, goza de un contenido básico que es admitido como lo mínimo esencial, un estándar a manera de componente objetivo que ninguna autoridad pública puede dejar de satisfacer. Este mismo autor argumenta que para dicho componente, objetivo del derecho a la vivienda, es necesario adoptar indicadores que puedan ser usados como parámetro para distinguir, por ejemplo, el incumplimiento de las obligaciones de un Estado o de particulares en el ámbito de los derechos habitacionales, o para establecer si una nación produjo, en un periodo determinado, situaciones de regresión o de progreso en la aplicación de la política de habitación. La importancia de los indicadores habitacionales está en ese papel de componente objetivo, para que acciones u omisiones del Estado, o de particulares, sean consideradas verdaderas violaciones del derecho a la vivienda.

El tránsito del morador a una vivienda con estándar residencial adecuado no necesariamente se asimila a un escenario con condiciones de habitabilidad. Aun cuando los indicadores de déficit habitacional nacionales incorporen los mínimos esenciales de la Guía de las Naciones Unidas de 1961, no se incluyen condiciones particulares de los contextos latinoamericanos ni necesidades particulares de población homogénea. Adicionalmente, el derecho a la vivienda ya 


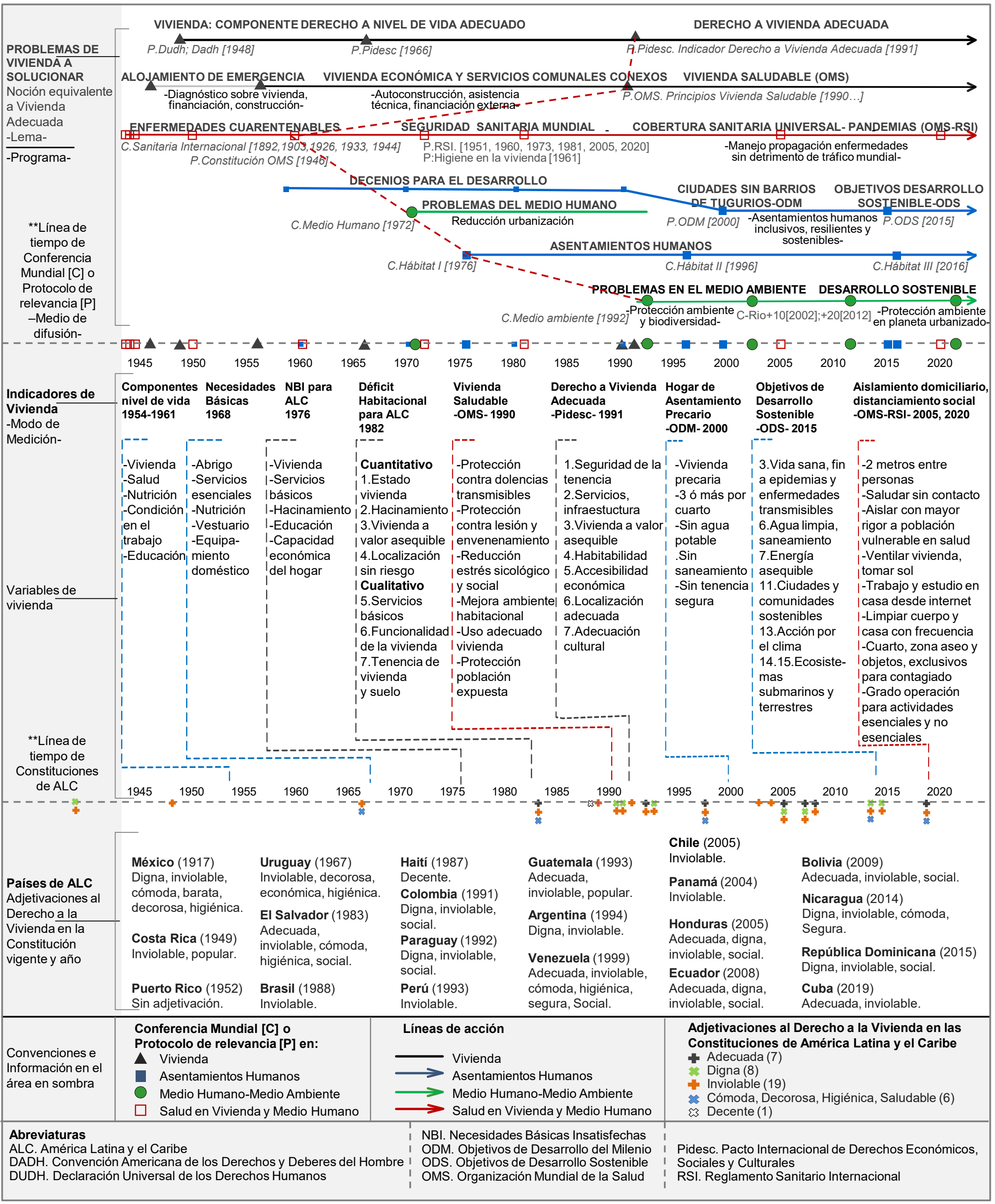

(4) Figura 2. Línea de tiempo sobre el discurso de las Naciones Unidas para acceso y provisión de vivienda adecuada financiarizada. 
ha sido atravesado por medidas financieras cuando desde su componente objetivo -las variables de habitación - no interpela el acceso a la vivienda y los servicios conexos desde instrumentos de financiación como impacto en la calidad de vida; en consecuencia, los procesos de financiamiento no existen como situación que podría violar el derecho a la vivienda, y así la garantía por la violación a este derecho es una vivienda o unos servicios conexos como riqueza, sin atender a la vivienda adecuada desde su condición de abrigo y lugar de desarrollo del ser humano.

El escenario de acceso y provisión de la vivienda según el ingreso familiar, desde las medidas de financiamiento, bien sea o no dentro del marco de la garantía del derecho a la vivienda, se corresponde con lo que expone Aalbers (2019) sobre financiarización, como

\section{[...] el creciente dominio de los actores financie- ros en los mercados, las prácticas, las mediciones y las narrativas a diversas escalas, que se traduce en una transformación estructural de las eco- nomías, las empresas (incluidas las instituciones financieras), los estados y los hogares [la traduc- ción es de la autora]. (p. 1)}

Lo enunciado también se corresponde con lo manifestado por Vergara-Perucich y Boano (2020), quienes aseguran que la financiarización de la vivienda se observa en la influencia de los actores financieros en los procesos de producción de la vivienda, en la relevancia de los instrumentos financieros en la comercialización de la vivienda y en el incremento de los actores que adquieren una vivienda con el fin de extraer valor de ella, más que para habitarla. Para adicionar como reflexión, también se aprecia en los productos habitacionales financiarizados que se entregan a quien ha sido lesionado por vivienda inadecuada, como garantía de los derechos habitacionales. Esta condición de la financiarización mantiene el escenario para la avalancha de desalojos por el no pago de créditos hipotecarios, de alquiler y de servicios conexos, además de ejecuciones hipotecarias; todos ellos, previstos en el Informe A/75/148 de 2020 de las Naciones Unidas sobre impactos de la COVID-19, y anunciados ampliamente en los noticieros mundiales en 2020.

\section{Conclusiones}

Las variables de los componentes del nivel de vida de 1961 configuran los indicadores de déficit cuantitativo y cualitativo de vivienda para los países de América Latina y el Caribe, y han sido constitutivas del Indicador de Asentamientos Precarios de los ODM a 2015, de manera disgregada de los ODS a 2030; además, se han visibilizado desde un marco de derechos cuando la vivienda fue considerada un componente del derecho a un nivel de vida adecuado en la
DUDH de 1948, en el PIDESC de 1966 y desde el derecho a la vivienda adecuada en el PIDESC de 1991. No obstante, las Naciones Unidas, desde la década de 1940 y entrado el siglo XXI, ha instituido un enfoque hacia la asequibilidad de la vivienda como problema a partir del ingreso; la preocupación por la calidad de vida no es evidente y confirma a la vivienda y los servicios conexos como servicios adquiridos mediante medios económicos, dentro del marco de políticas de habitación financiarizadas. Así, la vivienda adecuada es elegida por el morador a partir de decisiones sobre las variables de habitación que su ingreso económico puede cubrir; la capacidad económica definirán las inadecuaciones con las que se mora.

La vivienda que se habita no se convierte en inadecuada o insalubre por una situación que afecte la salud del morador, pero cuando la salud flaquea $y$ se requiere confinamiento en casa emerge la conciencia individual, colectiva y estatal, que el espacio en el cual se vive y donde se interactúa con otros influye en la integridad física y mental, y en el cuerpo recae la capacidad de resistir. También se tiene en consideración que el ingreso económico es lo que posibilita el acceso a las variables que configuran la vivienda adecuada y saludable, que pueden ayudar a minimizar los efectos del confinamiento; sin embargo, el aislamiento domiciliario genera un impacto devastador en los medios de subsistencia, lo que redunda en el acceso a la vivienda adecuada y sus servicios conexos, a los que se accede por el ingreso familiar.

Los Estados construyen el escenario al acceso a la vivienda financiarizada, que no siempre es adecuada, cuando la jurisprudencia de los países no configura objetivamente lo que es un derecho habitacional; cuando lo homologa a cuatro paredes y un techo y su garantía son las opciones limitadas en cuanto a habitabilidad que ofrece el mercado inmobiliario; cuando estipula variables mínimas de habitación no correspondientes a condiciones locales o culturales y desde el valor de la vivienda como abrigo; cuando no contempla que el acceso a la vivienda según el ingreso familiar y desde medidas financieras perturba la salud humana; cuando los ciudadanos no tienen a su alcance instrumentos jurídicos para exigir, en la práctica, el derecho a habitar una vivienda adecuada y saludable, que es más que el derecho a su acceso por subvenciones.

La garantía de la vivienda adecuada como derecho transita hoy entre la eficacia funcional, cuando desde la política habitacional se resarce la violación al derecho a una vivienda financiarizada, y su concepción social, cuando es considerada un servicio con acceso por ingreso, para la sobrevivencia del hombre y el abrigo a su integridad. 


\section{Referencias}

Aalbers, M. (2019). Financialization. International Encyclopedia of Geography: People, the Earth, Environment and Technology. doi:10.1002/9781118786352.wbieg0598. pub2.

https://onlinelibrary.wiley.com/doi/ abs/10.1002/9781118786352.wbieg0598

Academia de Ciencias de la Unión Soviética. (1967). Manual de economía política. Grijalbo.

Asamblea General de las Naciones Unidas. (1946). Resolución 53 (I). Vivienda y urbanismo. Organización de las Naciones Unidas. https://research.un.org/es/docs/ga/quick/ regular/1

Asamblea General de las Naciones Unidas. (1948). Resolución 217 (III). Carta Internacional de los Derechos del Hombre. Item A. Declaración Universal de los Derechos del Hombre. Organización de las Naciones Unidas.

https://documents-dds-ny.un.org/doc/ RESOLUTION/GEN/NRO/046/82/IMG/ NR004682.pdf?OpenElement

Asamblea General de las Naciones Unidas. (1955). A/2929. Annotations on the text of the draft International Covenants on Human Rights. Agenda Item 28. Part II. Annexes tenth session. Organización de las Naciones Unidas.

https://www2.ohchr.org/english/issues/ opinion/articles1920 iccpr/docs/A-2929.pdf

Asamblea General de las Naciones Unidas. (1960). Resolución 1508 (XV). Vivienda económica y servicios comunales conexos. Organización de las Naciones Unidas. https://documents-dds-ny.un.org/doc/ RESOLUTION/GEN/NR0/156/36/IMG/ NR015636.pdf?OpenElement

Asamblea General de las Naciones Unidas. (1961). Resolución 1676 (XVI). Urbanización. Organización de las Naciones Unidas. https://documents-dds-ny.un.org/doc/ RESOLUTION/GEN/NR0/170/98/IMG/ NR017098.pdf?OpenElement

Asamblea General de las Naciones Unidas. (1966). Resolución 2200 (XXI). Pacto Internacional de Derechos Económicos, Sociales y Culturales, Pacto Internacional de Derechos Civiles y Políticos y Protocolo Facultativo de Derechos Civiles y Políticos. Oganización de las Naciones Unidas.

https://documents-dds-ny.un.org/doc/ RESOLUTION/GEN/NR0/007/35/IMG/ NR000735.pdf?OpenElement

Asamblea General de las Naciones Unidas. (1976). Hábitat: Conferencia de las Naciones Unidas sobre los asentamientos humanos. Vancouver, 1976. A/CONF.70/15. Organización de las Naciones Unidas.

h t t ps://digitallibrary.un.org/ record/793768? In=es\#record-files-collapseheader

Asamblea General de las Naciones Unidas. (1996). Informe de la Conferencia de las Naciones Unidas sobre los Asentamientos Humanos (Hábitat II). A/CONF.165/14. Organización de las Naciones Unidas. h t t p s : //digitallibrary.un.org/ record/222703? In=es\# record-files-collapseheader
Asamblea General de las Naciones Unidas. (2000). Resolución 55/2. Declaración del Milenio. Organización de las Naciones Unidas. https://documents-dds-ny.un.org/ doc/UNDOC/GEN/N00/559/54/PDF/ N0055954.pdf?OpenElement

Asamblea General de las Naciones Unidas. (2015). Resolución 70/1. Transformar nuestro mundo: la Agenda 2030 para el DesarroIlo Sostenible. Organización de las Naciones Unidas.

https://documents-dds-ny.un.org/ doc/UNDOC/GEN/N15/291/93/PDF/ N1529193.pdf?OpenElement

Asamblea General de las Naciones Unidas. (2017). Nueva Agenda Urbana. Informe de la Conferencia de las Naciones Unidas sobre la Vivienda y el Desarrollo Urbano Sostenible (Hábitat III). Quito, 2016. A/CONF.226/12. Organización de las Naciones Unidas. h t t p s : //digitallibrary.un.org/ record/1289000? In=es\#record-files-collapseheader

Asamblea General de las Naciones Unidas. (2020). A/75/148. Informe del Relator Especial sobre una vivienda adecuada como elemento integrante del derecho a un nivel de vida adecuado y sobre el derecho de no discriminación a este respecto, Sr. Balakrishnan Rajagopal. La COVID-19 y el derecho a una vivienda adecuada: impactos y medidas futuras. Organización de las Naciones Unidas.

https://documents-dds-ny.un.org/doc/ UNDOC/GEN/N20/195/69/PDF/N2019569. pdf?OpenElement

Banco Mundial (1993). Housing: Enabling markets to work. Banco Mundial. https://elibrary.worldbank.org/doi/abs/10.1596/08213-2434-9

Comité de Derechos Económicos, Sociales y Culturales. (1991). El derecho a una vivienda adecuada (art. 11, párr. 1): 13/12/91. CESCR Observación general N. ${ }^{\circ} 4$ (General Comments). 6. ${ }^{\circ}$ período de sesiones (1991) (Vol. E/1992/23). Organización de las Naciones Unidas.

https://www.acnur.org/fileadmin/ Documentos/BDL/2005/3594.pdf

Comité de Derechos Económicos, Sociales y Culturales. (1997). El derecho a una vivienda adecuada, Observación general 7, (párrafo 1 del artículo 11 del Pacto), los desalojos forzosos. United Nations.

https://www.escr-net.org/es/recursos/ observacion-general-no-7-derecho-unavivienda-adecuada

Craven, M. (2003). History, pre-history, and the right to housing in international law. En S. Leckie (Ed.), National perspectives on housing rights (pp. 43-64). Martinus Nijhoff Publishers.

Le Corbusier. (1993). Principios de urbanismo (la Carta de Atenas). Planeta-Agostini.

Lefebvre, H. (1983). La revolución urbana. Alianza Editorial.

Mateos Jiménez, J. (2005). Actas de las Conferencias Sanitarias Internacionales (1851-1938). Rev Esp Salud Pública 79(3), 339-349. http://scielo.isciii.es/scielo.php?script =sci arttext\&pid=S1135-57272005000300003
Organización de las Naciones Unidas. (1961). Definición y medición internacional del nivel de vida. Guía provisional. Organización de las Naciones Unidas.

https://unstats.un.org/unsd/publication/ seriese/seriese_cn3_270_rev1s.pdf

Organización de las Naciones Unidas. (1972). Informe de la Conferencia de Naciones Unidas sobre el Medio Humano. Estocolmo. A/ CONF.48/14. Organización de las Naciones Unidas.

https://digitallibrary.un.org/record/523249? ln=es

Organización de las Naciones Unidas. (1992). Informe de la Conferencia de Naciones Unidas sobre el Medio Ambiente y el Desarrollo. Río de Janeiro. A/CONF.151/26. Organización de las Naciones Unidas.

https://digitallibrary.un.org/ record/160453? In=es

Organización Internacional del Trabajo. (1961). R.115-Recomendación sobre la vivienda de los trabajadores, 1961 (Núm. 115). Organización Internacional del Trabajo. https://www.ilo.org/dyn/normlex/ es/f?p=NORMLEXPUB:12100:0::NO::P12100 ILO_CODE:R115

Organización Mundial de la Salud. (1946). Constitución de la Organización Mundial de la Salud. Organización Mundial de la Salud. https://apps.who.int/gb/bd/PDF/bd48/ basic-documents-48th-edition-sp. pdf?ua $=1 \mathrm{\#}$ page $=7$

Organización Mundial de la Salud. (1961) Comité de expertos en higiene de la vivienda. Serie de informes técnicos N. ${ }^{\circ} 225$. Organización Mundial de la Salud. https://apps.who.int/iris/bitstream/ handle/10665/37728/WHO TRS 225 spa. pdf? sequence $=1$ \&isAllowed $=$ y

Organización Mundial de la Salud. (1990). Principios de higiene de la vivienda. Organización Mundial de la Salud.

https://apps.who.int/iris/bitstream/ handle/10665/38629/9243561278 spa. pdf?sequence $=1$ \&isAllowed $=y$

Organización Mundial de la Salud (2016). Reglamento Sanitario Internacional RSI 2005. Organización Mundial de la Salud. h t t p s: / / w w w. w h o. in t/ i h r / publications/9789241580496/es/

Pisarello, G. (2003). Vivienda para todos: un derecho en (de)construcción. El derecho a una vivienda digna y adecuada como derecho exigible. Icaria.

Rolnik, R. (2015). Guerra dos lugares: a colonização da terra e da moradia na era das finanças. Boitempo Editorial.

Vergara-Perucich, J. y Boano, C. (2020). El derecho a la ciudad financierizada. Bitácora Urbano Territorial, 30(3), 123-135. doi:10.15446/bitacora.v30n3.82437. 
A Portada: Catedral de Notre Dame de Paris durante los trabajos de reparación en octubre de 2020 Fotografía: Arquitecto Andrés Avila Gómez (octubre, 2020)
(A) Orientación editorial

\section{Enfoque y alcance}

La Revista de Arquitectura (Bogotá) ( (ISSN 1657-0308 Impresa y E-ISSN 2357-626X en línea) es una publicación científica seriada de acceso abierto, arbitrada mediante revisión por pares (doble ciego) e indexada, en donde se publican resultados de investigación originales e inéditos.

Está dirigida a la comunidad académica y profesional de las áreas afines a la disciplina. Es editada por la Facultad de Diseño y el Centro de Investigaciones (CIFAR) de la Universidad Católica de Colombia en Bogotá (Colombia).

La principal área científica a la que se adscribe la Revista de Arquitectura (Bogotá) según la OCDE es:

Gran área: 6. Humanidades

Área: 6.D. Arte

Disciplina: 6D07. Arquitectura y Urbanismo

También se publican artículos de las disciplinas como 2A02, Ingeniería arquitectónica; 5G03, Estudios urbanos (planificación y desarrollo); 6D07, Diseño.

Los objetivos de la Revista de Arquitectura (Bogotá) son:

- Promover la divulgación y difusión del conocimiento generado a nivel local, nacional e internacional

- Conformar un espacio para la construcción de comunidades académicas y la discusión en torno a las secciones definidas.

- Fomentar la diversidad institucional y geográfica de los autores que participan en la publicación.

- Potenciar la discusión de experiencias e intercambios científicos entre investigadores y profesionales.

- Contribuir a la visión integral de la arquitectura, por medio de la concurrencia y articulación de las secciones mediante la publicación de artículos de calidad.

- Publicar artículos originales e inéditos que han pasado por revisión de pares, para asegurar que se cumplen las normas éticas, de calidad, validez científica, editorial e investigativa.

- Fomentar la divulgación de las investigaciones y actividades desarrolladas en la Universidad Católica de Colombia.
Palabras clave de la Revista de Arquitectura (Bogotá): arquitectura, diseño, educación arquitectónica, proyecto y construcción, urbanismo.

Idiomas de publicación: español, inglés, portugués y francés.

Título abreviado: Rev. Arquit.

Titulo corto: RevArq

\section{Políticas de sección}

La revista se estructura en tres secciones correspondientes a las líneas de investigación activas y aprobadas por la institución, y dos complementarias, que presentan dinámicas propias de la Facultad de Diseño y las publicaciones relacionadas con la disciplina.

Cultura y espacio urbano. En esta sección se publican los artículos que se refieren a fenómenos sociales en relación con el espacio urbano, atendiendo aspectos de la historia, el patrimonio cultural y físico, y la estructura formal de las ciudades y el territorio.

Proyecto arquitectónico y urbano. En esta sección se presentan artículos sobre el concepto de proyecto entendido como elemento que define y orienta las condiciones proyectuales que devienen en los hechos arquitectónicos o urbanos, y la forma como estos se convierten en un proceso de investigación y nuevo de conocimiento. También se presentan proyectos que sean resultados de investigación, los cuales se validan por medio de la ejecución y transformación en obra construida del proceso investigativo. También se contempla la publicación de investigaciones relacionadas con la pedagogía y didáctica de la arquitectura, el urbanismo y el diseño.

Tecnología, medioambiente y sostenibilidad. En esta sección se presentan artículos acerca de sistemas estructurales, materiales y procesos constructivos, medioambiente y gestión, relacionados con los entornos social-cultural, ecológico y económico.

Desde la Facultad. En esta sección se publican artículos generados en la Facultad de Diseño, relacionados con las actividades de docencia, extensión, formación en investigación o internacionalización, las cuales son reflejo de la dinámica y de las actividades realizadas por docentes, estudiantes y egresados; esta sección no puede superar el $20 \%$ del contenido.

Textos. En esta sección se publican reseñas, traducciones y memorias de eventos relacionados con las publicaciones en Arquitectura y Urbanismo.
A Frecuencia de publicación

Desde 1999 y hasta el 2015, la Revista de Arquitectura (Bogotá) publicó un volumen al año, a partir del 2016 se publicarán dos números por año en periodo anticipado, enero-junio y julio-diciembre, pero también maneja la publicación anticipada en línea de los artículos aceptados (versión Post-print del autor).

La Revista de Arquitectura (Bogotá) se divulga mediante versiones digitales (PDF, HTML, EPUB, XML) e impresascon un tiraje de 700 ejemplares, los tiempos de producción de estas versiones dependerán de los cronogramas establecidos por la editorial.

Los tiempos de recepción-revisión-aceptación pueden tardar entre seis y doce meses dependiendo del flujo editorial de cada sección y del proceso de revisión y edición adelantado.

Con el usuario y contraseña asignados, los autores pueden ingresar a la plataforma de gestión editorial y verificar el estado de revisión, edición o publicación del artículo.
(A) Canje

La Revista de Arquitectura (Bogotá) está interesada en establecer canje con publicaciones académicas, profesionales o científicas del área de Arquitectura y Urbanismo, como medio de reconocimiento y discusión de la producción científica en el campo de acción de la publicación.

Mecanismo

Para establecer canje por favor descargar, diligenciar y enviar el formato: RevArq FP20 Canjes

Universidad Católica de Colombia
(202I, enero-junio). Revista de
Arquitectura (Bogotá), 23(I),
I-I24. Doi: 10.14718

ISSN: I657-0308
E-ISSN: 2357-626X
Especificaciones:
Formato: $34 \times 24 \mathrm{~cm}$
Papel: Mate II5 g
Tintas: Negro y policromía

Contacto

Dirección postal:

Avenida Caracas No. 46-72

Universidad Católica de Colombia

Bogotá D. C., Colombia

Código postal: 111311

Facultad de Diseño

Centro de Investigaciones (CIFAR)

Sede El Claustro. Bloque "L", 4 piso

Diag. 46A No. 15b-10

Editor, Arq. César Eligio-Triana

Teléfonos:

+57 (1) $3277300-3277333$

Ext. 3109; 3112 o 5146

Fax: +57 (1) 2858895
Correo electrónico:

revistadearquitectura@ucatolica.edu.co cifar@ucatolica.edu.co

Página WEB:

www.ucatolica.edu.co

Vínculo Revistas científicas

http://publicaciones.ucatolica.edu.co revistas-cientificas

https://revistadearquitectura.ucatolica.edu.co/ 
Reflexiones en torno a la enseñanza de la arquitectura y el urbanismo en Colombia. Conversaciones con Juan Carlos

m. Pérgolis Valsecchi, René Carrasco Rey y Juan Carlos del

¿ Castillo

Reflections on the teaching of architecture and urban planning in Colombia. Conversations with Juan Carlos Pérgolis Valsecchi, René Carrasco Rey y Juan Carlos del Castillo

웅

La experiencia de caminar en ciudades latinoamericanas

The experience of walking in Latin American cities

Movilidad urbana de la población en la ciudad de Encarnación * Paraguay. Desarrollo urbano y gestión ambiental

$m$ Urban mobility of the population in the city of Encarnación, Paraguay.

这

ษั

La vivienda adecuada financiada según el ingreso.

El discurso de las Naciones Unidas

பं

La arquitectura frente a las innovaciones pedagógicas.

Pervivencia y resignificación de la Escuela Nueva en el Cono Sur Architecture facing the pedagogical innovations. Survival and resignification of the New School in the Southern Cone

Aporte de las competencias investigativas a la integración de saberes curriculares.

Una visión en el escenario de aprendizaje del diseño

arquitectónico

Contribution of research competencies to the integration of curricula knowledge. A vision in the architectural design learning scenario

Adobe como saber ancestral usado en construcciones

N autóctonas de Pore y Nunchía, Casanare (Colombia)

j. Adobe as an ancestral knowledge used in autochthonous constructions $\stackrel{4}{\longleftarrow}$

Paisaje construido y sustentabilidad urbana: huellas

$œ$

El Plan de Transformación de Osorno

Built landscape and urban sustainability: Identity prints of the modern landscape. The Osorno Transformation Plan

Indicadores de sostenibilidad social y su relación con el

$\hat{a}$

ن்

노 caso de estudio en Cuenca (Ecuador)

ن

Confort térmico en vivienda social multifamiliar de clima cálido en Colombia

느 Thermal comfort in multi-family social housing in a warm climate in ن் Colombia
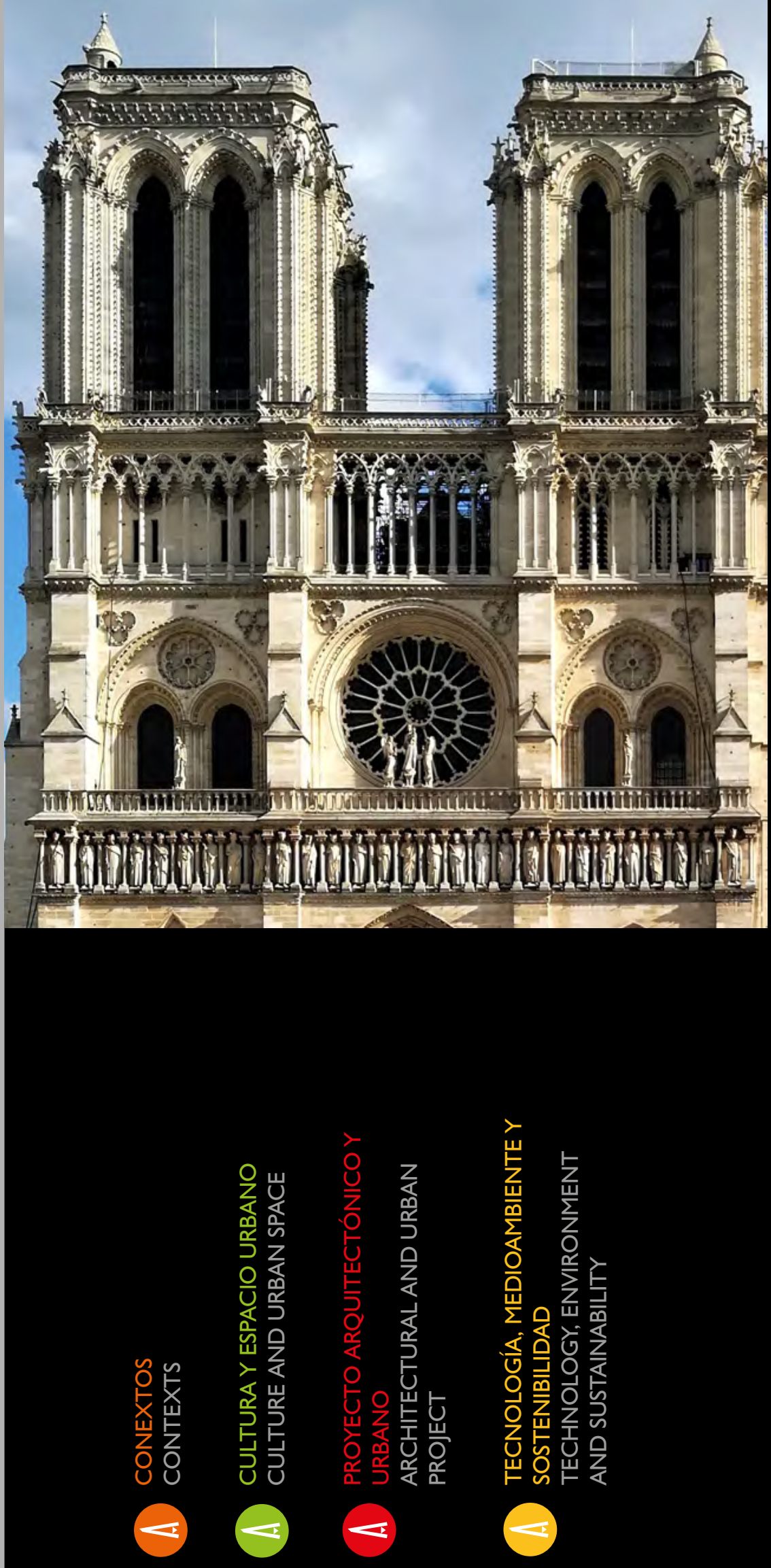

La Revista de Arquitectura es de acceso abierto, arbitrada e indexada y está presente en:
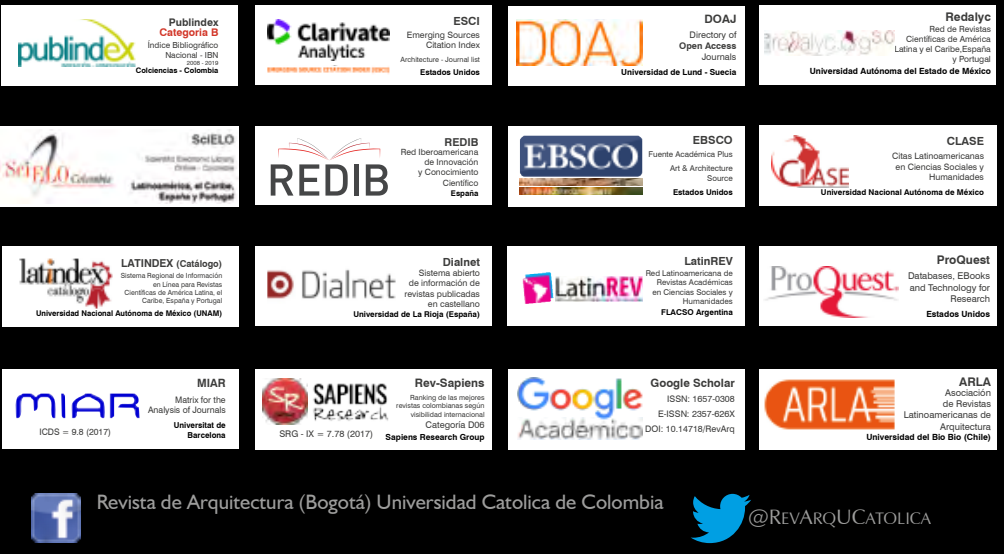

8
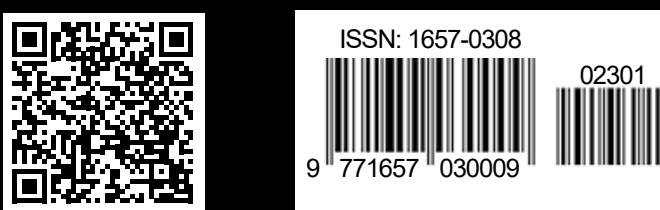